\title{
Towards a framework for analyzing determinants of performance of community health workers in malaria prevention and control: a systematic review
}

Helen Mwiinga Chipukuma ${ }^{*}$, Joseph Mumba Zulu², Choolwe Jacobs ${ }^{3}$, Gershom Chongwe ${ }^{3}$, Mumbi Chola $^{3}$, Hikabasa Halwiindi ${ }^{4}$, Jessy Zgambo ${ }^{3}$ and Charles Michelo ${ }^{3}$

\begin{abstract}
Background: Community health workers (CHWs) are an important human resource in improving coverage of and success to interventions aimed at reducing malaria incidence. Evidence suggests that the performance of CHWs in malaria programs varies in different contexts. However, comprehensive frameworks, based on systematic reviews, to guide the analysis of determinants of performance of CHWs in malaria prevention and control programs are lacking.

Methods: We systematically searched Google Scholar, Science Direct, and PubMed including reference lists that had English language publications. We included 16 full text articles that evaluated CHW performance in malaria control. Search terms were used and studies that had performance as an outcome of interest attributed to community-based interventions done by CHWs were included.

Results: Sixteen studies were included in the final review and were mostly on malaria Rapid Diagnosis and Treatment, as well as adherence to referral guidelines. Factors determining performance and effective implementation of CHW malaria programs included health system factors such as nature of training of CHWs; type of supervision including feedback process; availability of stocks, supplies, and job aids; nature of work environment and reporting systems; availability of financial resources and transport systems; types of remuneration; health staff confidence in CHWs; and workload. In addition, community dynamics such as nature of community connectedness and support from the community and utilization of services by the community also influenced performance. Furthermore, community health worker characteristics such marital status, sex, and CHW confidence levels also shaped CHW performance.

Conclusions: Effectively analyzing and promoting the performance of CHWs in malaria prevention and control programs may require adopting a framework that considers health systems and community factors as well as community health worker characteristics.
\end{abstract}

Keywords: Community health worker, Performance, Evaluation, Malaria, Assessment, Utilization, Implementation, Policy

\footnotetext{
* Correspondence: hchipukum@yahoo.co.uk

${ }^{1}$ Department of Health Policy Unit, School of Public Health, University of

Zambia, P.O. Box 50110, Lusaka, Zambia

Full list of author information is available at the end of the article
}

(c) The Author(s). 2018 Open Access This article is distributed under the terms of the Creative Commons Attribution 4.0 International License (http://creativecommons.org/licenses/by/4.0/), which permits unrestricted use, distribution, and reproduction in any medium, provided you give appropriate credit to the original author(s) and the source, provide a link to the Creative Commons license, and indicate if changes were made. The Creative Commons Public Domain Dedication waiver (http://creativecommons.org/publicdomain/zero/1.0/) applies to the data made available in this article, unless otherwise stated. 


\section{Background}

Globally, malaria has declined in incidence by $37 \%$ and mortality rate by $60 \%$ between 2000 and 2015 [1]. Community interventions through Community Health Workers (CHWs), who are a link between the community and the health facility, have contributed to this reduction [2-4]. The World Health Organization (WHO), in attempting to move toward elimination of malaria, has come up with a strategy that has three main building blocks which are to ensure universal access to malaria prevention, diagnosis, and treatment, to accelerate efforts toward elimination of malaria and attainment of malaria-free status, and to transform malaria surveillance into a core intervention [2]. These building blocks can be best achieved through Primary Health Care (PHC) using the $\mathrm{CHW}$ as key actors in the strategy, which most countries adopted after the 1978 Declaration of Alma-Ata [3, 4].

Community health workers are men and women with basic literacy and numeracy levels, chosen by the community and trained to deal with individual and community health problems while working in close relationship with the formal health care system [5-7]. This review focused on trained CHWs who work as community malaria agents (CMAs) carrying out malaria prevention and control interventions in the community.

Studies have shown that CHW performance can help reduce morbidity and mortality in resource constrained settings $[3,7,8]$. The CHW performance is contextspecific [7], and there is little evidence on what specific factors have contributed to effective implementation of the CHW strategy in malaria interventions. Community health workers provide cost-effective and sustainable ways of delivering malaria control interventions in the community. These interventions include conducting rapid diagnostic tests, malaria treatment, community sensitization for IRS, and distribution of insecticide treated nets (ITNs) [9]. Despite great efforts to combat it, the threat of resurgent malaria is present across different settings. Resurgence has in part been attributed to non-cooperation of communities in control initiatives [10]. Awareness of this threat and the development of systems to minimize resurgence are key to further progress in malaria control [10]. This systematic review was done to gather evidence of CHW performance evaluations in malaria and how they were measured, highlighting determinants of their performance in malaria prevention and control programs.

\section{Methods}

\section{Search strategy}

Google Scholar, Science Direct, and PubMed were searched from September to October 2017. We also searched for and retrieved articles from reference lists using different terms for community health workers. Search terms included "Community Health Workers" OR
"Community Malaria Agents" OR "Community Based Volunteers" OR "Village Health Workers" OR "Community Health Aides" OR "Community Health Agents" "Health Extension Workers" OR "Health Surveillance Assistants" OR "Community Medicine Distributors" AND malaria AND "evaluation" OR "assessment" OR "performance" AND "Sub-Sahara Africa". Alternative terms for performance were evaluation and assessment.

\section{Inclusion criteria}

The search was limited to English peer-reviewed publications of observational and interventional studies with quantitative and mixed methods analysis. The review included publications addressing evaluation or performance assessment outcomes of $\mathrm{CHWs}$ working in malaria preventive and curative programs in the sub-Sahara Africa. Only publications from 2000 to 2016 were included, as this marked the period in which there was a distinct drop in malaria cases and deaths attributed to $\mathrm{CHW}$ efforts. Studies that evaluated CHW programs which covered effectiveness of $\mathrm{CHWs}$ or responsiveness of individual $\mathrm{CHWs}$, community and program implementers to the malaria CHW strategy with regards to sensitization, surveillance, diagnosis, treatment, follow-up of malaria cases, environmental management, and described a factor promoting or affecting malaria $\mathrm{CHW}$ program outcomes were included.

\section{Study selection and quality assessment}

Four reviewers from a team of eight independently assessed titles and abstracts. The other four reviewers read the full texts of identified peer-reviewed articles to evaluate potential eligibility. Another reviewer's opinion was sought in case of persisting disagreements until consensus was reached. The study selection was guided by the PRISMA guidelines, and quality of these studies was assessed using the critical appraisal skills program (CASP 2015) to ensure methodically proven reliable evidence-based studies in the review. The quality criteria we used were as follows:

- Whether the research questions or objectives were clearly stated?

- Whether the approach was appropriate for the research question?

- Whether the study context was clearly described?

- Whether the role of the researcher was clearly described?

- Whether the sampling method was clearly described?

- Whether the sampling strategy was appropriate for the research question? 
- Whether the method of data collection was clearly described?

- Whether the data collection method was appropriate to the research question?

- Whether the method of analysis was clearly described?

- Whether the analysis was appropriate for the research question?

- Whether the claims made are supported by sufficient evidence?

All studies included in the review focused on malaria CHW strategy with clearly stated objectives addressing the question under review and highlighting factors that affected performance positively or negatively. The sampling methods were well explained, and the data collection methods were described clearly with appropriate analyses whose claims were supported by evidence in all the articles.

Data was extracted onto a data extraction form created in Microsoft Excel to assess information on key study aspects such as the objectives, designs, sample, performance measurement tool, and results. The data extraction form also contained a description of the intervention of study and the outcome measures (Table 1).

\section{Data analysis and synthesis}

Data from the selected articles was analyzed using NVivo version 10 software. The analysis involved identification, coding, and exploration of relationships of themes within data. A code list was developed which comprised of broad themes collectively agreed upon by the research team members after preliminary reading of abstracts. The code list was later modified to accommodate emergent themes and imported into NVivo. Data from the included articles was coded in the respective nodes by two separate researchers including the principal investigator to allow for intercoder reliability tests. Where there were discrepancies, the researchers discussed until consensus was reached on how information could be coded. Code reports were for identification of specific factors affecting performance of $\mathrm{CHW}$ in malaria programs. Descriptive analysis of the contents of all papers reviewed was conducted per category (thematic coding) and new (sub) categories deriving from the literature were added to the framework (Table 2).

\section{Results}

The search resulted in a total of $N=1692$ results appearing on the databases of which $n=251$ were from Google Scholar, $n=796$ from Science Direct, $n=619$ from PubMed, and $n=26$ from reference lists of some articles. After title screening, $n=613$ articles remained and $n=527$ duplicates were removed leaving $n=86$ articles for full abstract assessment. Abstracts not reporting the outcome of interest were $n=44$, and $n=42$ were considered for full text review. No full texts were found for $n=19$ articles, and $n=7$ were excluded as they were establishing use of $\mathrm{CHW}$ for an intervention while others were establishing efficacy of antimalarial drug given by CHWs. Some papers were excluded because they were not from sub-Saharan Africa and were published outside the review period. All the search results were managed in Endnote referencing software (Thomson Reuters, Philadelphia, USA) and the final 16 articles have been reported in this systematic review (Fig. 1).

\section{Study characteristics}

A total of 16 studies are included in this review, 5 of which were from East Africa, 4 from Central Africa, 3 from West Africa, and 4 from Southern Africa. Countries represented were Uganda with four studies [1114], Kenya with three [15-17], one from Rwanda [18], one from Tanzania [19], one from Burkina Faso [20], one from Ghana [21], one from Mali [22], one from Malawi [23], and three from Zambia [24-27]. Twelve of the studies had a cross-sectional design [11-18, 20, $22,23,25]$, one was a mixed methods prospective study [24], one was a cluster randomized control trial [26], one was a randomized crossover trial [22], and one randomized control stepped wedge trial [21] (Table 1).

\section{Outcome of malaria evaluations}

Most studies included in this review were evaluating diagnosis and treatment in community case management representing $88 \%$ of the studies that evaluated performance [11-14, 16, 18, 21-24, 26, 28]. Performance outcomes in malaria varied in different contexts, though generally poor. For instance, some deficiencies were found in management of sick children in Integrated Community Case Management (ICCM) [11, 16] despite reports that $\mathrm{CHWs}$ were able to use rapid diagnostic test (RDT) and artemisinin-based combination therapy (ACT) $[19,24,26]$ and that the strategy has potential for universal coverage [23]. Different factors perceived to influence performance were grouped into individual $\mathrm{CHW}$ characteristics, health system factors, and community factors (Table 2). Only two studies evaluated adherence which was generally high while referral practices were poor [21, 24]. Two of the included studies assessed general performance in relation to the outcome indicators based on general scheduled activities $[15,18]$. Table 1 gives a summary of included studies indicating the study designs, method of evaluation, 


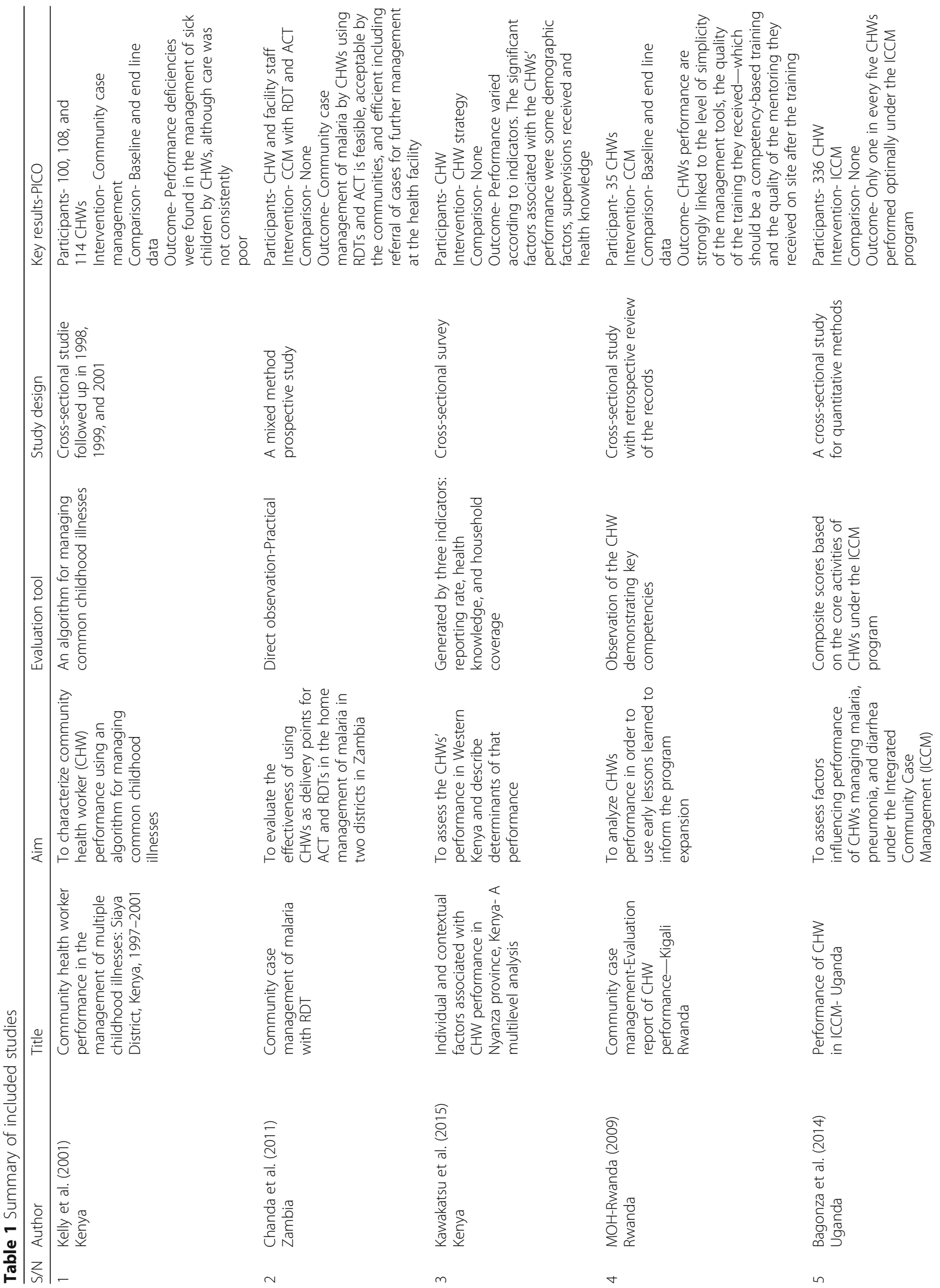




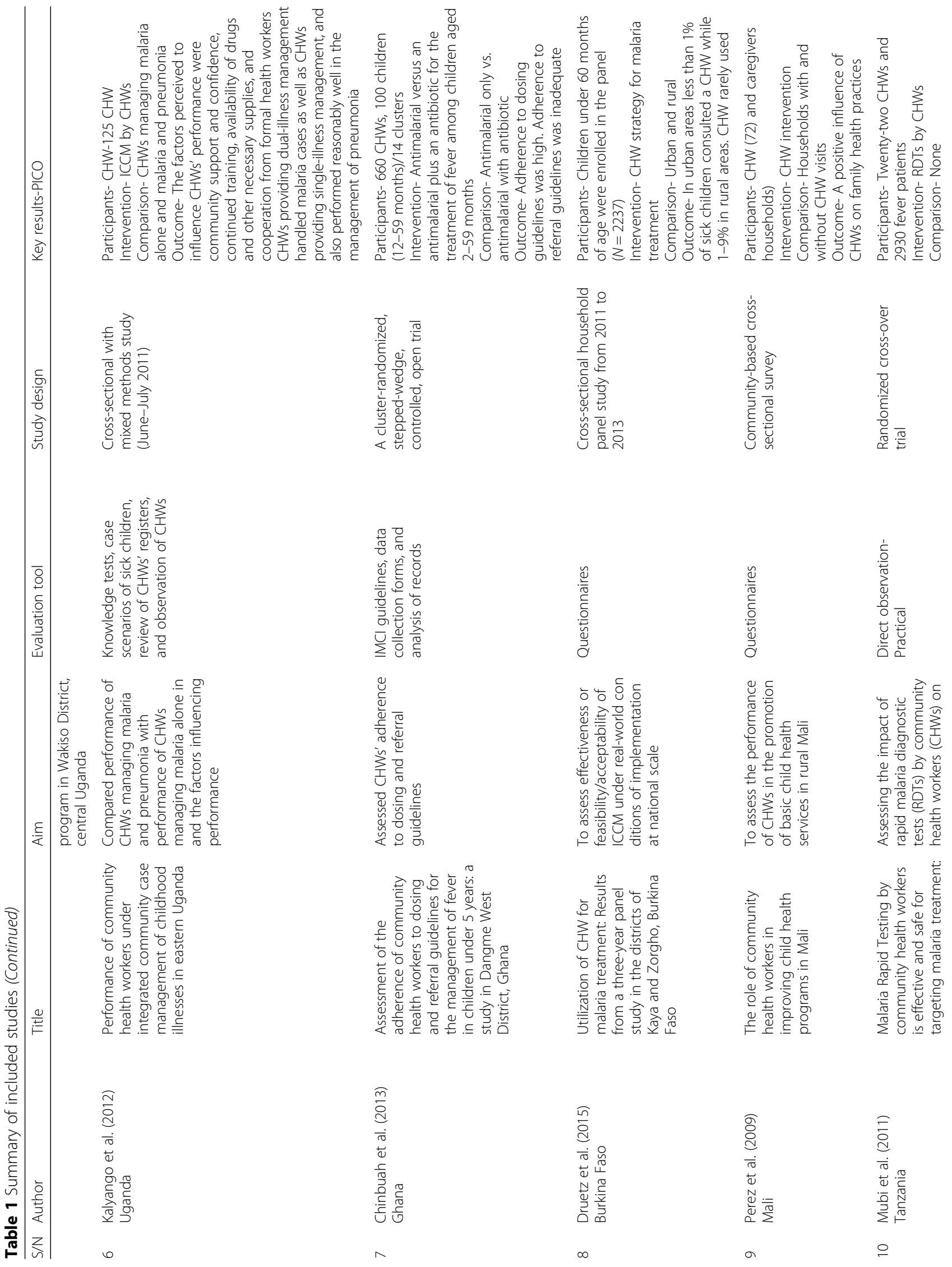




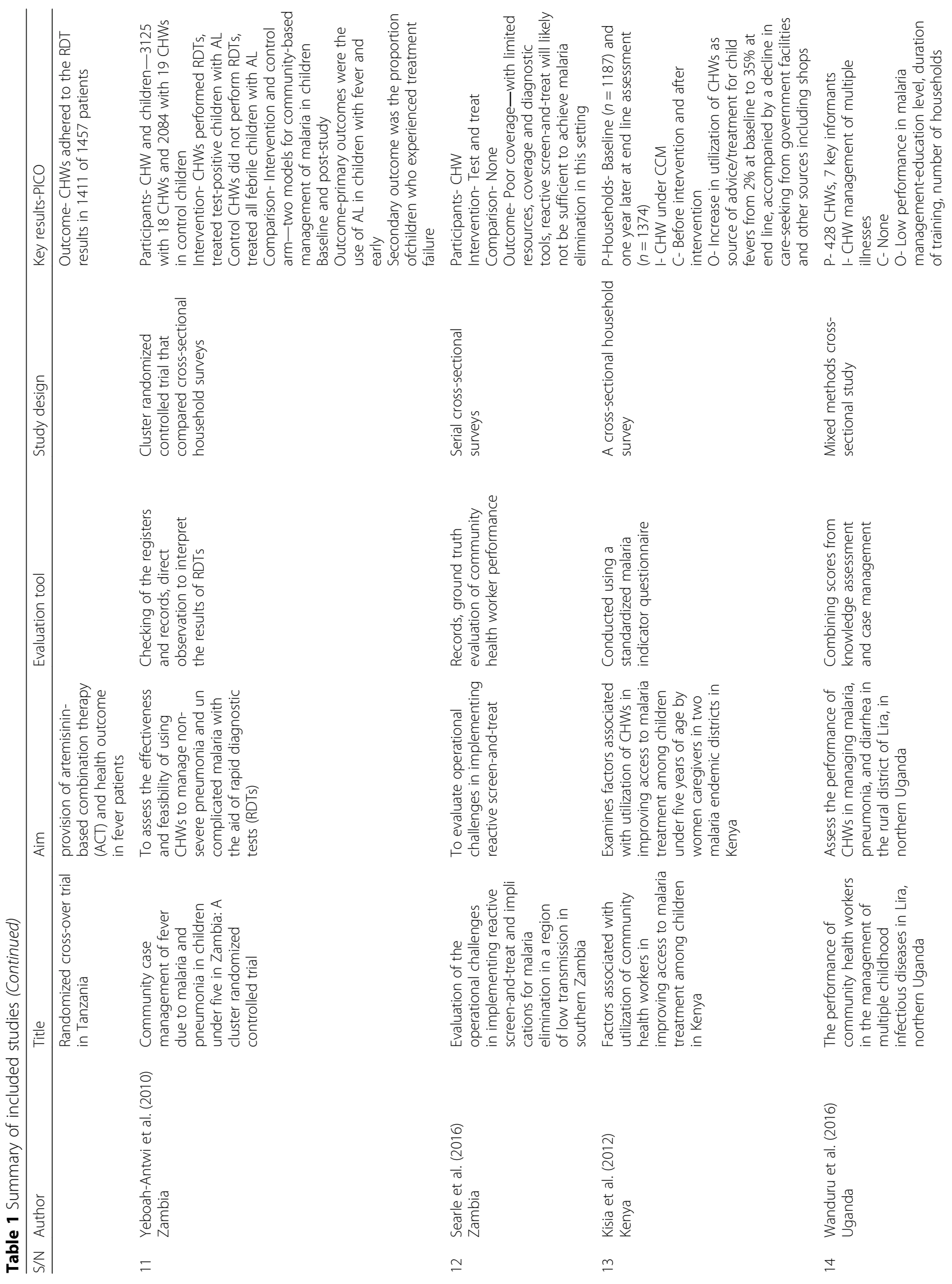




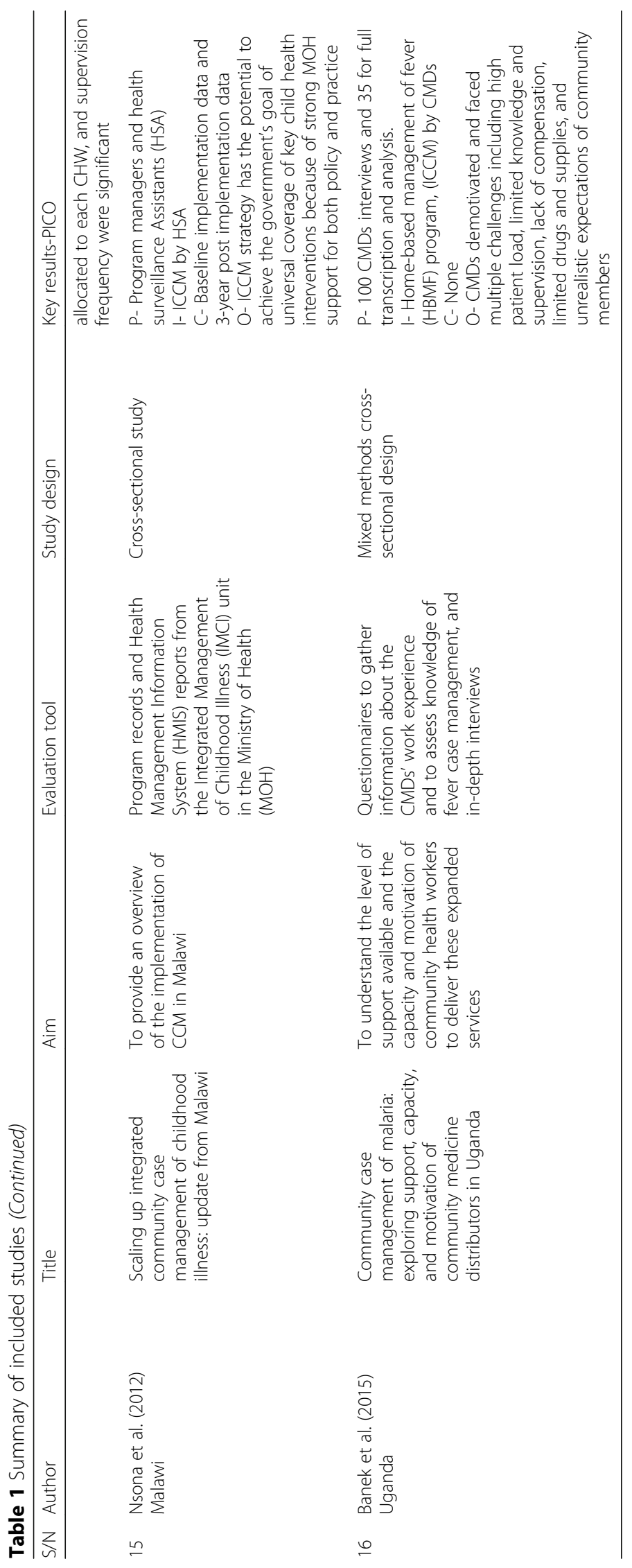


Table 2 Determinants of performance in malaria programs

\begin{tabular}{ll}
\hline $\mathrm{S} / \mathrm{N}$ Factor $\quad$ Description of determinant & Studies \\
\hline Community health worker $(\mathrm{CHW})$ characteristics &
\end{tabular}

1 Demographic factors

2 CHW confidence and competence

\section{Community factors}

1 Community factors

2 CHW service utilization

\section{Health system factors}

1 Feedback

$2 \quad$ Training

$3 \quad$ Stocks and supplies

$4 \quad$ Job aids

5 Supervision

$6 \quad$ Funding

$7 \quad$ Transport

8 Remuneration/motivation

$9 \quad$ Health professional support

10 Workload
Female CHWs performed better than their male counterparts, married CHWs gave a higher performance than others, having fewer household duties encourages CHWs to work more actively and reduces the dropout rate, longer work experience, good educational status, availability of supporters for household chores, and appropriate sanitation practices

CHWs may have lacked confidence in the guidelines, particularly, the ability of $\mathrm{CHWs}$ to obtain an accurate history of convulsions. CHW able to do RDT confidently and effectively on the other hand

Mobilization of communities by the local leaders and confidence of the community in medicines used, lack of community appreciation for age restrictions of children treated, poor community participation, poor cooperation from caregivers, social prestige, community support in terms of feedback and rewards, training institute, poor performance of basic household health practices

$\mathrm{CHWs}$ rarely use for malaria interventions and only poor household using them in mostly in rural

Some CHWs did not receive timely feedback from their supervisors. Community support in the form of feedback and rewards

Adequately continuously trained and appropriately resourced CHWs are really motivated to perform their tasks like interpreting RDTs, and give treatment for Malaria. clearly defined roles for CHAs and supervisors

The need for continuous supplies of drugs and stocks is cardinal for enhancing success of CHWs for malaria

Complexity of guidelines was an important reason for deficiencies managing sick children. These should be in local language possibly

High quality support supervision from supervisors from formal health system who should have adequate health knowledge to conduct routine supervisions to sustain a high performance is necessary to improve the performance of $\mathrm{CHWs}$ in malaria interventions

CHW performance is hard to achieve and to maintain without sufficient consideration for funding

Distance and lack of transport refund affects their performance

Lack of incentives demotivates them and CHWs asking for consideration

\section{staff}

CHWs performed poorly due to large population coverage and multiple tasks
Kawakatsu et al. 2015; Bagonza et al. 2014; Wanduru et al. 2016

Kelly et al. 2001; Mubi et al. 2011

Kalyango et al. 2012; Druetz et al. 2015; Chinbuah et al. 2013; Kawakatsu et al. 2015; Perez et al. 2009; Banek et al. 2015; Nsona et al. 2012

Druetz et al. 2015; Kisia et al. 2012

Kelly et al. 2001; Yeboah-Antwi et al. 2010

Bagonza et al. 2014; Kalyango et al. 2012

Kalyango et al. 2012; Chanda et al. 2011;

MOH-Rwanda 2009; Yeboah-Antwi et al. 2010;

Druetz et al. 2015; Perez et al. 2009; Nsona et al.

2012; Wanduru et al. 2016

Kelly et al. 2001; Chanda et al. 2011; Kalyango et al. 2012; Searle et al. 2016; Druetz et al. 2015; Perez et al. 2009; Chinbuah et al. 2013; Banek et al. 2015; Nsona et al. 2012

Chinbuah et al. 2013; Kelly et al. 2001; Nsona et al. 2012

Bagonza et al. 2014; Kawakatsu et al. 2015; Perez et al. 2009; Kelly et al. 2001; Druetz et al. 2015; Chinbuah et al. 2013; Nsona et al. 2012; Wanduru et al. 2016

Druetz et al. 2015; Banek et al. 2015; Nsona et al. 2012

Kalyango et al. 2012; Perez et al. 2009

Druetz et al. 2015; Perez et al. 2009; Kalyango et al. 2012; Chinbuah et al. 2013; Banek et al. 2015; Nsona et al. 2012; Wanduru et al. 2016

Kalyango et al. 2012

Yeboah-Antwi et al. 2010; Kalyango et al. 2012; Searle et al. 2016; Perez et al. 2009; Kelly et al. 2001; Bagonza et al. 2014; Wanduru et al. 2016 
Table 2 Determinants of performance in malaria programs (Continued)

\begin{tabular}{llll}
\hline S/N & Factor & Description of determinant & Studies \\
\hline 11 & Evaluation environment & $\begin{array}{l}\text { Evaluated in a different setting other than area } \\
\text { of usual practice }\end{array}$ & Kelly et al. 2001 \\
& CHW performance may have been underestimated \\
because a failure to document was interpreted & Reporting & Kelly et al. 2001; Nsona et al. 2012 \\
& Poor CHW program coordination at all levels & Nsona et al. 2012 \\
13 & Program coordination & affects performance negatively & \\
\hline
\end{tabular}

sample characteristics, intervention, comparison, and the outcome of the study (PICO).

\section{Factors shaping the performance of community health workers in malaria prevention interventions}

This section outlines the factors that shape the performance of CHWs in programs aimed at managing malaria. The factors have been grouped into three major headings: CHW characteristics, health systems factors, and community factors. Table 2 gives details of different factors influencing performance of $\mathrm{CHW}$ in malaria interventions.

\section{Community health worker characteristics Demographic factors}

Being a female $\mathrm{CHW}$ was associated with performance as some female community members who were found home during $\mathrm{CHW}$ visits felt freer to be served by a fellow female CHW [11]. Being married also showed to have an influence on performance due to availability of supporters within the household of the CHWs to enable him or her perform her malaria-related duties [11, 15]. Attainment of secondary-level education positively influenced performance as CHWs would easily read and write monthly reports [14]. Longer work experience also

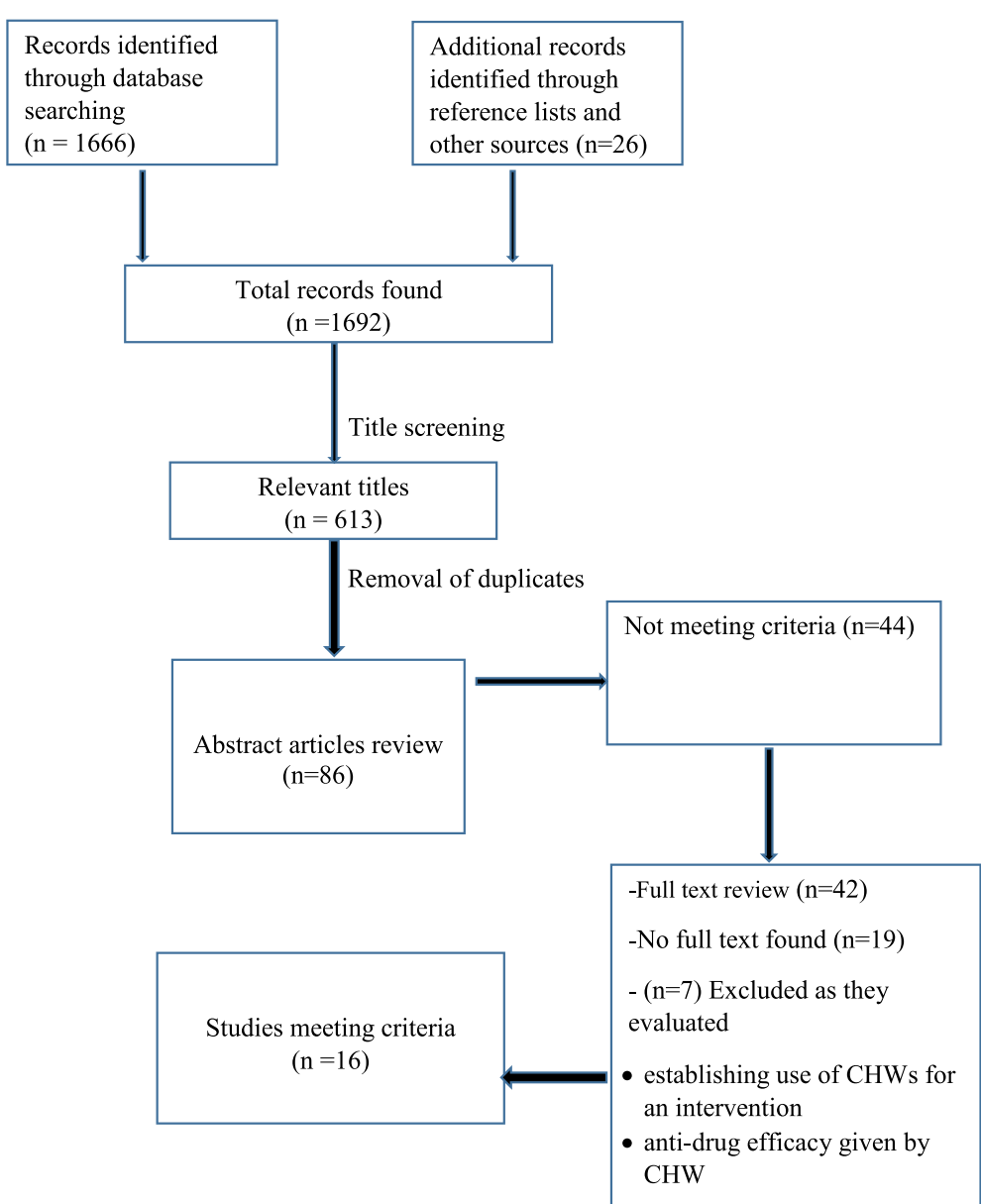

Fig. 1 Flow chart: search results 
positively influenced performance as it provided an opportunity to receive effective training, supervision, and incentives and to build a confidential relationship with community members [15].

\section{Competence and confidence levels}

How well the CHWs were conversant with the guidelines for Integrated Management of Childhood Illnesses (IMCI) guidelines was vital in promoting work performance. CHWs who lacked knowledge in the guidelines could not perform certain tasks such as obtaining an accurate history of convulsions in successive evaluations due to different terms used that may have meant shivering or startles hence recording poor performance [16]. Competence and confidence in undertaking tasks was considered in four studies investigating $\mathrm{CHW}$ ability to use RDT for malaria diagnosis and treatment in the community $[19,24,26,28]$. Results showed that CHWs were able to manage malaria fevers correctly and that RDTs in the hands of CHWs may safely improve early and well-targeted ACT treatment in malaria patients at community level in Africa. Following guidelines was a key determinant of competence and confidence in one's responsibilities [16].

\section{Health system factors}

\section{Availability of financial resources}

Poor performance in Community Case Management (CCM) was partly due to inadequate funding which negatively affected CHW work motivation as CHWs could not meet the set targets [13, 20, 23]. In Burkina Faso, for instance, less than $10 \%$ of the overall funding to scale up interventions against malaria was allocated to CCM, despite the ambitious objective to have CHWs manage $80 \%$ of all simple malaria cases. This goes to confirm the statement, "there has been no serious attempt to globalize investment in CHWs as a strategy to combat malaria" [20].

\section{Motivation}

Eight studies emphasized on the importance of motivation for enhancing CHW performance [12, 13, 16, 20-23, 27]. Motivation of CHWs with adequate support from the health system through the introduction of financial incentives and remuneration were among key factors to improve the work of CHWs in rural-urban area communities [13, 20, 22]. Rewards such as t-shirts, blood pressure checks, and transport fares remunerated during review meetings were also found to have greater influence on $\mathrm{CHW}$ performance [12, 21]. The CHWs were, in several programs, employed on a voluntary basis or as a regular employee without a fixed monthly salary [22]. Studies have recommended that local health authorities and community representatives clarify how CHWs will be employed. This has a bearing on their motivation to perform tasks, feasibility of sustaining community health activities, as well as affecting efforts of decreasing dropout rates of CHWs to avoid additional costs of replacing them [15, 22]. In Malawi, the stability and community earned respect of the Health Surveillance Assistants (HSAs) has been attributed to their recognition as formal members of the health work force. Provision of adequate housing to HSAs in hard-to-reach areas through village health committees also helped in improving performance, as well as raising the social status of the CHWs and their families [13, 16, 23].

\section{Transport systems}

Community health workers may live in places too far for community members to consult them [20]. Lack of transport for following up treated children and delivering malaria tasks is another hindrance to work performance $[12,22]$. In most cases, CHWs cover vast areas, which complicate their ability to effectively follow up children in rural communities [12, 22].

\section{Training}

Nine studies indicated the importance of training of CHW prior to implementing malaria interventions $[12,14,18,20,22-24,26,27]$. These studies show that adequately, continuously trained and appropriately resourced CHWs are motivated to perform their tasks such as interpreting RDTs, and giving treatment for malaria [26]. This is in addition to having clear definition and an understanding of staff responsibilities at the health posts [27]. Evidence shows that $\mathrm{CHW}$ trainings must be short as they perform better with only introduction to basic concepts in relation to managing diseases and are to take 2-3 days. Longer training may lead to information overload and thus result in less retention [23]. Performance is strongly linked to the level of simplicity of the management tools including the quality of training received. The training should be a competency-based training focused on exercises, demonstration, role play, video projection, case studies, and practices. Performance is also linked to the quality of mentoring they received on site after training [18]. The CHWs were trained only to prescribe antimalarial drugs to confirmed malaria cases and refer other non-malaria patients to the nearest health facility for further management. They also received training in filling in registers, managing drug supplies, counseling caregivers of children, and adverse reaction monitoring $[12,24]$. Some settings also showed that CHWs faced obstacles to performance due to lack of $\mathrm{CHW}$ training or regular refresher courses [20,27]. 


\section{Supervision process}

High quality support and supervision by supervisors from the formal health system is necessary to improve $\mathrm{CHW}$ performance. The ability of the supervisor to effectively translate knowledge acquired from the supervisory course into proper definition of tasks at the health posts was essential in enhancing performance [11, 15, $16,20-23,27]$.

Insufficient supervision affected the performance of CHWs. For instance, in some supervisory records, fewer than half of CHWs who performed poorly had received one-on-one clinical supervision at a health facility in the past year and a few others had not received any supervision in the previous 6 months $[16,20,22]$. The CHWs who had met with their supervisors in the last 3 months were likely to perform better than those who had not [27], and performance improvement was noticed when trained field supervisors provided additional support and fortnight supervision [21].

Another aspect of supervision is feedback processes. Two studies emphasized the importance of providing feedback in shaping work performance in the ICCM $[11,12,27]$. However, nearly a half of the CHWs did not receive timely feedback from their supervisors [11]. Regular supervision and CHW involvement in meetings were important because it provided opportunities for interactions, clarifications, and receiving feedback, which can act as a social glue for holding staff together [27].

\section{CHW program coordination}

Strong links between community programs and the formal health system are required to ensure appropriate training and supervision, and adequate remuneration of community health workers but these components still remain weak [23]. There must be clear leadership at central (Ministry of Health), provincial, and district levels and an understanding of stakeholders' roles and responsibilities under WHO guidelines. These are to monitor and evaluate programs and develop appropriate evaluation tools, reporting tools, and registers. Quality of care assessment of CHWs performance is important as well as districtcommunity-clinic review meetings to strengthen implementation of CHW programs for malaria [23].

\section{Reporting System}

Only two studies $[16,23]$ highlighted issues concerning reporting $\mathrm{CHW}$ performance. The two studies indicated that performance may have been underestimated because failure to document could have been interpreted as an error-missing data [16]. Performance and efficiency may be improved with innovations such as provision of mobile phones to CHWs. This would facilitate contact and SMS-based reporting and for logistics management information systems to strengthen use and management of medicines and other supplies [16, 23].

\section{Availability of stocks and supplies}

The need for continuous supply of stock is cardinal for motivation and enhancing success of CHWs [12, 16, 20-25]. Following training, the health facility should provide logistics and supplies for the CHWs' routine work [24, 25]. Some studies indicated poor performance due to CHW program factors such as irregular supply or lack of materials to enable them to perform their work at night and during rainy weather [12]. Insufficient RDTs reported by over $50 \%$ of $\mathrm{CHWs}$ sometimes were as a consequence of high number of cases during the peak malaria season [25] and difficulty in anticipating additional quantities of RDTs needed to conduct reactive case detection. This highlights the need for guidance on quantification of medicines to District Medical Officers [23]. Provision of medicines and supplies to CHWs by supervisors during their monthly visits may help to alleviate stock outs [23]. Other hindrances included the lack of aides such as watches and treatment guidelines. The treatment guidelines were reported to be long and that they had ambiguities in the clinical algorithm, and they also showed discrepancies between the drug dosing chart and the algorithm [16].

\section{Nature of health professional support}

The CHWs expressed concern about criticism from health professional staff for unnecessarily referring a child to a health facility. As a result, when CHWs are in doubt, they may tend to choose a less severe classification or decide not to recommend referral even when they assign a severe classification [16]. Sometimes CHWs would refer a patient to the health center but they would not get the needed attention as the health workers displayed mistrust of the referral instead of attending to the patient [12].

\section{Nature of the evaluation environment}

The unfamiliar hospital setting also made some CHWs nervous, leading to errors [16]. Although they were instructed to provide treatment as though they were in their home community, some CHWs did not follow the procedures correctly in the hospital environment and could not also document the need for referral [16]. As a result the evaluation which was conducted in the hospital setting showed that some referral data were missing [16].

\section{Amount of workload}

Seven studies discussed workload issues as affecting work performance $[11,12,14,16,22,25,26]$. The 
CHWs performed poorly due to large population coverage and multiple tasks. They tend to get overwhelmed with so many programs when they are to be apportioned only a reasonable amount of work. Workload was mentioned as a key determinant of performance in qualitative analysis [14]. For example, poor basic household health practices put pressure on CHWs for regular follow-up [11, 12, 22]. Thus follow-ups were difficult due to high number of cases during peak malaria season $[22,25]$. In contrast, in terms of multitasking, two studies concluded that with appropriate training, adequate supervision, provision of drugs and necessary supplies, community support, and feedback provision from the formal health system, CHWs can provide integrated treatment for malaria and pneumonia, and that additional tasks do not reduce the quality of malaria community case management $[11,12,16,26]$.

\section{Community factors \\ Support from the community and community connectedness}

Eight studies reported community factors and their role in shaping work performance [12, 13, 15, 20-23, 27]. Community ownership through dialog before introduction of the services is vital for successful participation in the malaria programs, for instance, formation of village health committees ( $\mathrm{VHC}$ ) and engagement of community leaders to manage the VHC [23]. Factors affecting performance included mobilization of communities by local leaders and confidence of the community in medicines used by CHWs. Community support in the form of feedback and rewards such as in-kind incentives from community members was found to have greater influence on CHW performance than that from the health system [12, 27]. Some settings have faced some of the most common obstacles to performance, such as caregivers resisting to be being referred $[20,21]$ and unrealistic expectations of caregivers [13]. Social prestige and community support are the important community level factors associated with CHW performance [15].

\section{Utilization of services provided by CHWs}

Another factor affecting performance related to how the community is utilizing the services provided by $\mathrm{CHWs}$ $[16,17,20,26]$. Certain skills such as referral by CHWs in some areas could not be meaningfully evaluated because of the small number of clients $[16,20]$. People chose a different source of treatment other than CHWs because of various reasons, but distance was statistically significant $[20,26]$. Poor and smaller community size of less than 100 households were important characteristics influencing the utilization of CHW services as community case management was offered for free and that CHWs provided prompt treatment at household level
[17]. This suggests that issues related to implementation fidelity, acceptability, or feasibility have undermined the effectiveness of $\mathrm{CHW}$ programs in countries like Burkina Faso [20].

\section{Discussion}

This systematic review has found that CHW performance was evaluated differently in different malaria endemic settings without a standard evaluation tool. Most evaluations were competency based, focusing mainly on RDT, treatment, and referral services. This is similar to a study by Yasauko (2010) that assessed the quality of service of village malaria workers in Cambodia. In this study, village health workers (VHW) focused on diagnosis and treatment, ignoring other community malaria preventive roles. The study recommended the need to cover other aspects of malaria control in order to further strengthen community-based malaria control [29]. The roles need to include an integrated approach covering surveillance, communication, vector control, and environmental management including evaluating implementation of these instilled $\mathrm{CHW}$ skills in terms of quality and a measure of the intended purpose of the intervention to the community as CHW performance cannot only be evaluated through a skill but also by an outcome of the intervention [30].

Using evidence of determinants of performance in malaria interventions, we have proposed a performance framework which shows the elements that are vital for determining performance in community malaria programs. The major components are health system factors, CHW characteristics, and community factors (Fig. 2).

Our findings indicate that all elements of the WHO building blocks within the health system interlink in a complex manner and may not flow in a linear manner all the time. These have a significant influence on implementation of $\mathrm{CHW}$ programs in malaria interventions and their performance. The individual CHW characteristics are modified by the health system factors to create an enabling environment for individual CHWs, while community factors also play a role in shaping overall performance (Fig. 2). This means that CHWs cannot implement community malaria interventions without support from the formal health system. According to the findings, the malaria $\mathrm{CHW}$ strategy needs considerations for $\mathrm{CHW}$ characteristics like selecting at least more female CHWs, married CHWs, those with longer experience in community service, secondary level educational status, those with availability of support for household chores, and those with good sanitation practices. These characteristics should be taken into account when developing the selection criteria for $\mathrm{CHW}$ community-based malaria programs. The community leadership must be involved and consulted in 


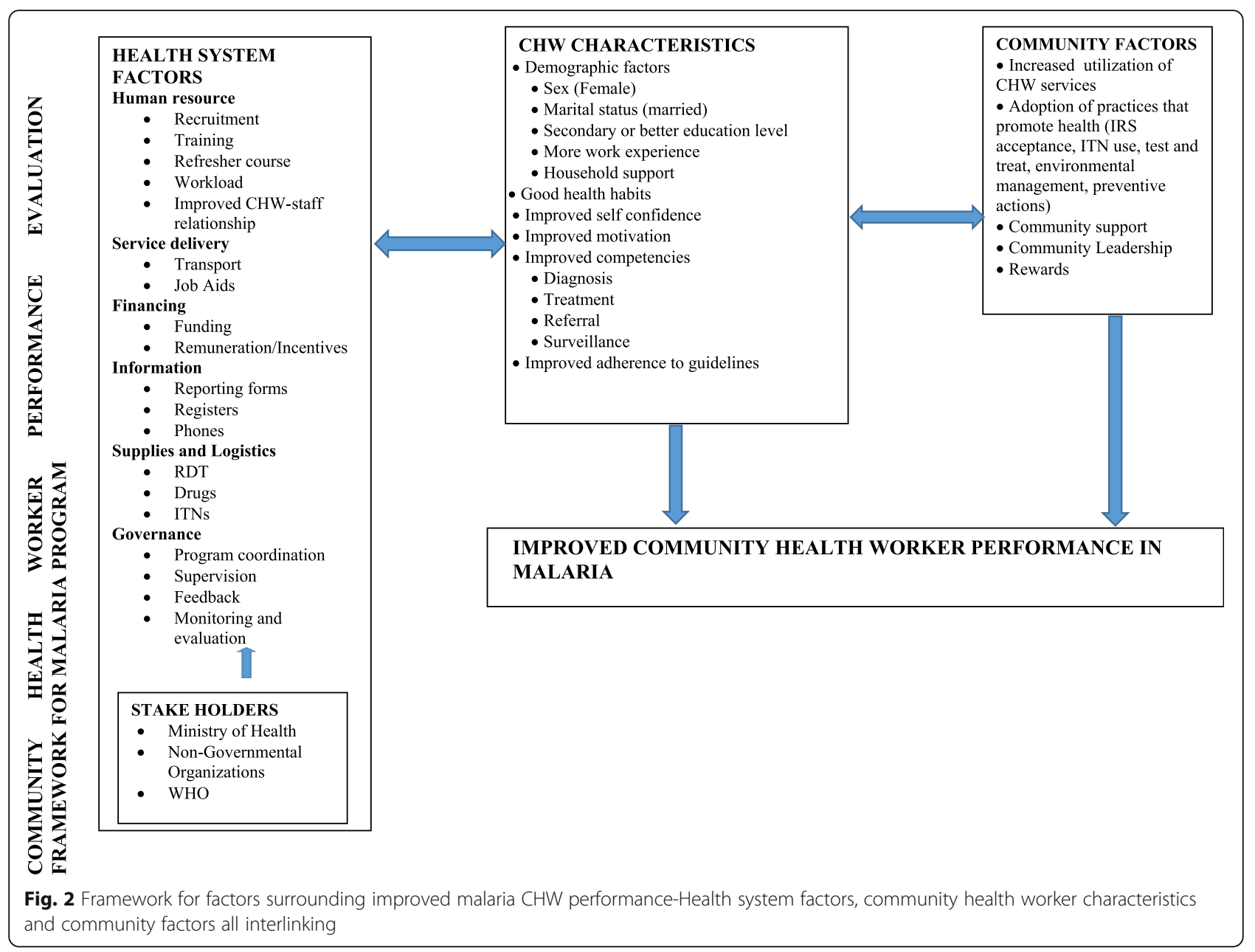

selecting which CHWs may be engaged as this may assist in obtaining positive response from the community to the malaria interventions [7]. In addition, while WHO recommends that $\mathrm{CHW}$ should receive remuneration, most CHWs are working on voluntary basis and their working terms are not made clear to them except for example the Malawian government that has salaried the health surveillance assistants including providing accommodation, a system that has led to improvement and scaling up of ICCM [23].

Improvement of $\mathrm{CHW}$-professional staff relationship is important for improving $\mathrm{CHW}$ confidence, as it provides a level of motivation for a well-coordinated $\mathrm{CHW}$ program. Improved cognitive reflections exhibited in CHWs are part of performance measurements worth considering [30]. The $\mathrm{CHW}$ competencies in diagnosis, treatment, referral, and surveillance are a measure of individual $\mathrm{CHW}$ performance in malaria, but output is also dependent on training received, refresher courses, supervision, logistics, and supplies. Evidence indicates that competence evaluations through observations may have better results if evaluations are done within the $\mathrm{CHW}$ work environment [16]. A systematic review by Smith et al. 2014 on the effectiveness of strategies to improve community case management (CCM) of malaria reports that CHWs are able to provide good quality malaria care, including performing procedures such as rapid diagnostic tests with appropriate training, clear guidelines, and regular supportive supervision [31]. Evidence from a systematic review on the impact and implementation of supervision suggests that improving supervision quality has a greater impact than increasing frequency of supervision alone [32].

There is a need to strengthen CHW program coordination among stakeholders such as health ministries and nongovernmental organizations that play a big role in strategy or intervention implementation at district level. These stakeholders have a direct influence on the health system factors related to production of guidelines, registers, checklists, reporting tools, and evaluation tools of which studies revealed not to have had reporting or standard evaluation tools for malaria programs [11, 12, 15]. However, this review found that there are few or no 
reporting or standard evaluation tools for malaria programs though an innovation to improve the information system through use of phones for reporting has been effected in some countries like Zambia [33].

Motivated CHW may help encourage the community to adopt practices that promote health through witnessing visible changes in the community by $\mathrm{CHW}$ efforts as they are a link to the community [7]. Remuneration, availability of supplies, and relevant infrastructure have been found to be important demotivating factors for health workers [34]. To avoid demotivating $\mathrm{CHW}$ and health workers alike, sufficient remuneration; supplies of RDT, drugs, and ITNs; and job aids need to be consistent, including relevant infrastructure [23, 27, 34]. One study indicates that there is no serious attempt to globalize investment in CHWs as a strategy to combat malaria [35] hence funding allocation and remuneration for CHW programs should be increased if malaria elimination is to be achieved [20,23,35].

In this review, CHWs performed poorly due to increased workload as they have a large population coverage and perform multiple tasks. There is need for scaling up of these malaria CHW interventions and promoting continued use of CHWs in national programs as an important human resource that contributes to longterm impact of interventions $[4,35]$. Integrating malaria control activities for CHWs as a holistic package is critical in the fight to eradicate malaria [27]. This entails continued recruitment and training to help reduce the workload and increase coverage. A systematic review assessing the most effective approach to delivering malaria treatment in developing countries recommended that $\mathrm{CHWs}$ roles should be recognized, integrated, and expanded as they were effective despite challenges met [35]. Adequate training and refresher courses are vital as knowledge on malaria epidemiology and vector ecology is essential in promoting integration and expansion of CHW practice [36]. This integrated approach may help foster the new WHO pillar strategy that has three main building blocks which are to ensure universal access to malaria prevention, diagnosis, and treatment, to accelerate efforts toward elimination of malaria and attainment of malaria-free status, and to transform malaria surveillance into a core intervention [2]. However, integrating and multitasking with other health programs needs total health system support as priority for CHWs to perform as an important cadre in delivery of primary health care services to the community [4].

The CHWs serve the community, and community feedback is therefore vital as this influences performance. Studies included in the review indicated poor utilization of CHW services [20] and that only the rural poor are utilizing their services [17]. Utilization of CHWs can be improved through engagement of community leadership who may influence the community to support the $\mathrm{CHW}$ malaria strategy through rewards [17] and adopting practices that prevent and control malaria. The CHW program is intended not only to improve intervention coverage but also to improve surveillance and reduce congestion in health facilities even in urban areas which are densely populated [3].

\section{Strengths and limitations}

The strength of this review is that it shows actual determinants of $\mathrm{CHW}$ performance specific to community malaria interventions in the sub-Saharan Africa region. It adds value to current literature, as it included both qualitative and quantitative studies and was able to explore perceptions. Additionally, this review reflects performance evaluation areas that have been previously poorly explored with respect to $\mathrm{CHW}$ interventions in malaria programs. Emphasis has been on improved competencies in diagnosis, treatment, and referral, concentrating on factors within the $\mathrm{CHW}$ sphere that enhance performance but are a measure of individual performance in itself. Despite these findings, this systematic review may have been limited by language restriction to English only and also by CHWs having different names in different settings, possibly leading to exclusion of some eligible studies.

\section{Conclusion}

This review has shown that health system factors, community factors, and CHW characteristics were important factors that affect $\mathrm{CHW}$ performance with the health system factors being the most important. These CHWs are available and willing to serve the community but a workable environment for them has not been well established in many health systems. Factors affecting performance highlighted in this review should be taken into account by policymakers during designing, implementation, and adjusting of $\mathrm{CHW}$ programs with consideration that these factors interlink. Using evidence of performance determinants in malaria programs from various studies, we therefore suggest that a $\mathrm{CHW}$ performance framework developed from these studies could guide designing, implementation, and evaluation of community-based malaria prevention programs.

\section{Abbreviations}

ACT: Artemisinin-based combination therapy; CBV: Community-based volunteers; CHA: Community health aides OR Community health agents; CHW: Community health workers; CMA: Community malaria agents; CMD: Community medicine distributor; HEW: Health extension workers; HSA: Health surveillance assistants; ICCM: Integrated community case management; IRS: Indoor residual spraying; ITN: Insecticide treated net; PHC: Primary health care; RDT: Rapid diagnostic test; VHW: Village health workers 


\section{Acknowledgments}

Helen Mwiinga Chipukuma/H.M.C. is a recipient of a TDR scholarship under the Postgraduate Training Scheme in Implementation Research at the University of Zambia. We are grateful to the financial support for the training scheme as provided by the UNICEF/UNDP/World Bank/WHO Special Program for Research and Training in Tropical Diseases (TDR). I am also grateful to my husband Kelvin Mwiinga for his encouragement and unending academic support.

\section{Availability of data and materials}

Data is included in the manuscript

\section{Authors' contributions}

HMC conceived the review and did the literature search, analysis, and reporting. The data was extracted by $H M C, J M Z$, and CJ. JMZ refined the title, aided in drafting manuscript, and structured the review. JMZ, CJ, GC, JZ, $H H, M C$, and CM reviewed and edited the manuscript for intellectual content. All authors contributed to analysis and interpretation of data. The opinions expressed are those of authors alone. All authors read and approved final manuscript.

\section{Authors' information}

The main author, Helen Mwiinga Chipukuma (hchipukum@yahoo.co.uk), is a Public Health Masters graduate from the University of Zambia, School of Public Health in the Department of Health Policy and Management with implementation research.

\section{Ethics approval and consent to participate}

Not applicable

\section{Competing interests}

The authors declare that they have no competing interests.

\section{Publisher's Note}

Springer Nature remains neutral with regard to jurisdictional claims in published maps and institutional affiliations.

\section{Author details}

'Department of Health Policy Unit, School of Public Health, University of Zambia, P.O. Box 50110, Lusaka, Zambia. ${ }^{2}$ Department of Health Promotion Unit, School of Public Health, University of Zambia, P.O. Box 50110, Lusaka, Zambia. ${ }^{3}$ Department of Epidemiology and Biostatistics Unit, School of Public Health, University of Zambia, P.O. Box 50110, Lusaka, Zambia. ${ }^{4}$ Department of Environmental Health Unit, School of Public Health, University of Zambia, P.O. Box 50110, Lusaka, Zambia.

\section{Received: 30 May 2017 Accepted: 19 April 2018}

\section{Published online: 08 May 2018}

\section{References}

1. WHO. Regional profiles 2015. Geneva: World Health Organization; 2015.

2. WHO. World malaria report 2015. Geneva: World Health Organization; 2015.

3. Pallas SW, et al. Community health workers in low-and middle-income countries: what do we know about scaling up and sustainability? Am J Public Health. 2013;103(7):e74-82.

4. Christopher JB, et al. Thirty years after Alma-Ata: a systematic review of the impact of community health workers delivering curative interventions against malaria, pneumonia and diarrhoea on child mortality and morbidity in sub-Saharan Africa. Hum Resour Health. 2011;9:27.

5. Lehman U, Sanders D. Community health workers: what do we know about them? The state of the evidence on programmes, activities, costs and impact on health outcomes of using community health workers. Geneva: World Health Organization; 2007. p. 1-42.

6. Zulu JM, et al. Developing the national community health assistant strategy in Zambia: a policy analysis. Health Res Policy Syst. 2013;11(24). https://doi.org/10.1186/1478-4505-11-24.

7. WHO. Community health workers: what do we know about them? Geneva: WHO Department of Human Resources for Health; 2007.

8. Haines A, et al. Achieving child survival goals: potential contribution of community health workers. Lancet. 2007;369(9579):2121-31.
9. Yasuoka J, et al. Scale-up of community-based malaria control can be achieved without degrading community health workers' service quality: the village malaria worker project in Cambodia. Malar J. 2012;11:4.

10. Cohen JM, et al. Malaria resurgence: a systematic review and assessment of its causes. Malar J. 2012;11:122

11. Bagonza J, Kibira S, Rutebemberwa E. Performance of community health workers managing malaria, pneumonia and diarrhoea under the community case management programme in central Uganda: a cross sectional study. Malar J. 2014;13(1):367.

12. Kalyango JN, et al. Performance of community health workers under integrated community case management of childhood illnesses in eastern Uganda. Malar J. 2012;11:282.

13. Banek K, et al. Community case management of malaria: exploring support, capacity and motivation of community medicine distributors in Uganda. Health Policy Plan. 2015;30(4):451-61.

14. Wanduru $P$, et al. The performance of community health workers in the management of multiple childhood infectious diseases in Lira, northern Uganda-a mixed methods cross-sectional study. Glob Health Action. 2016;9:33194.

15. Kawakatsu $Y$, et al. Individual and contextual factors associated with community health workers' performance in Nyanza Province, Kenya: a multilevel analysis. BMC Health Serv Res. 2015;15(1):1.

16. Kelly JM, et al. Community health worker performance in the management of multiple childhood illnesses: Siaya District, Kenya, 1997-2001. Am J Public Health. 2001;91(10):1617-24.

17. Kisia J, et al. Factors associated with utilization of community health workers in improving access to malaria treatment among children in Kenya. Malar J. 2012;11:248

18. $\mathrm{MOH}$-Rwanda. Community $\mathrm{IMCl} /$ community case management: evaluation report of community health workers performance. Kigali: Rwanda Ministry of Health; 2009. http://www.basics.org/documents/community-IMCI\%20and-Community-Case-Management-Evaluation_Rwanda.pdf. Accessed 21 Dec 2015.

19. Mubi M, et al. Malaria rapid testing by community health workers is effective and safe for targeting malaria treatment: randomised cross-over trial in Tanzania. PLoS One. 2011;6(7):e19753.

20. Druetz T, et al. Utilization of community health workers for malaria treatment: results from a three-year panel study in the districts of Kaya and Zorgho, Burkina Faso. Malar J. 2015;14:71.

21. Chinbuah MA, et al. Assessment of the adherence of community health workers to dosing and referral guidelines for the management of fever in children under 5 years: a study in Dangme West District, Ghana. Int Health. 2013:5(2):148-56.

22. Perez F, et al. The role of community health workers in improving child health programmes in Mali. BMC Int Health Hum Rights. 2009;9(1):28.

23. Nsona $\mathrm{H}$, et al. Scaling up integrated community case management of childhood illness: update from Malawi. Am J Trop Med Hyg. 2012;87(5 Suppl):54-60.

24. Chanda $\mathrm{P}$, et al. Community case management of malaria using ACT and RDT in two districts in Zambia: achieving high adherence to test results using community health workers. Malar J. 2011;10:158.

25. Searle KM, et al. Evaluation of the operational challenges in implementing reactive screen-and-treat and implications of reactive case detection strategies for malaria elimination in a region of low transmission in southern Zambia. Malar J. 2016;15(1):412.

26. Yeboah-Antwi K, et al. Community case management of fever due to malaria and pneumonia in children under five in Zambia: a cluster randomized controlled trial. PLoS Med. 2010;7(9):e1000340.

27. Zulu JM, et al. Hope and despair: community health assistants' experiences of working in a rural district in Zambia. Hum Resour Health. 2014;12(1):30.

28. Counihan $\mathrm{H}$, et al. Community health workers use malaria rapid diagnostic tests (RDTs) safely and accurately: results of a longitudinal study in Zambia. Am J Trop Med Hyg. 2012:87(1):57-63.

29. Yasuoka J, et al. Assessing the quality of service of village malaria workers to strengthen community-based malaria control in Cambodia. Malar J. 2010;9:109.

30. Kok MC. Performance of community health workers: optimizing the benefits of their unique position between communities and the health sector. 'sHertogenbosch: Uitgeverij Box Press; 2015.

31. Smith Paintain $L$, et al. Community health workers and stand-alone or integrated case management of malaria: a systematic literature review. Am J Trop Med Hyg. 2014;91(3):461-70. 
32. Hill Z, et al. Supervising community health workers in low-income countries - a review of impact and implementation issues. Glob Health Action. 2014;7(1):24085.

33. Hamainza B, et al. Comparison of a mobile phone-based malaria reporting system with source participant register data for capturing spatial and temporal trends in epidemiological indicators of malaria transmission collected by community health workers in rural Zambia. Malar J. 2014;13:489.

34. Thu NTH, Wilson A, McDonald F. Motivation or demotivation of health workers providing maternal health services in rural areas in Vietnam: findings from a mixed-methods study. Hum Resour Health. 2015;13(1):91

35. Kamal-Yanni MM, Potet J, Saunders PM. Scaling-up malaria treatment: a review of the performance of different providers. Malar J. 2012;11:414

36. Lim S, et al. Promoting community knowledge and action for malaria control in rural Cambodia: potential contributions of village malaria workers. BMC Res Notes. 2012:5:405.

Ready to submit your research? Choose BMC and benefit from:

- fast, convenient online submission

- thorough peer review by experienced researchers in your field

- rapid publication on acceptance

- support for research data, including large and complex data types

- gold Open Access which fosters wider collaboration and increased citations

- maximum visibility for your research: over $100 \mathrm{M}$ website views per year

At BMC, research is always in progress.

Learn more biomedcentral.com/submissions 